\title{
Renal Cell Carcinoma: A Cancer-testis Antigen Poor Malignancy
}

\author{
Sepideh Faramarzi, Soudeh Ghafouri-Fard
}

Department of Medical Genetics, Shahid Beheshti University of Medical Sciences, Tehran, Iran.

\begin{abstract}
Renal cell carcinoma ( $\mathrm{RCC}$ ) is a relatively frequent cancer with increasing incidence in some regions. There is a need for early diagnosis of this cancer as a significant number of patients develop metastasis in their clinical course. Cancer-testis antigens (CTAs) are a group of tumor associated antigens whose expression has been assessed in a wide range of malignancies. CTAs are also considered as immunotherapy targets. Considering the relative responsiveness of RCC patients to immunotherapy, expression analysis of CTAs in RCC is of clinical importance. However, data regarding expression of CTAs in RCC is scarce except for a few numbers of CTAs including NYESO-1. The expression pattern of CTAs in RCC samples and cell lines is reviewed in this manuscript.
\end{abstract}

Keywords: Renal cell carcinoma- cancer-testis antigen- biomarker- immunotherapy

Asian Pac J Cancer Biol, 2 (3), 63-66

\section{Introduction}

Renal cell carcinoma (RCC) is among frequent cancers in the world with rising incidence in developing countries. Patients' outcome is considerably better in localized cancers compared with cases which have local and distant metastasis, therefore early diagnosis of cancer is of practical significance [1]. Although surgery is recommended for localized RCCs, metastatic RCCs are not cured with this kind of therapy. Consequently, several immunotherapeutic approaches are suggested for metastatic RCCs. Administration of cytokines has been effective for only a fraction of highly selected patients. Targeted agents such as vascular endothelial growth factor tyrosine kinase inhibitors and mammalian target of rapamycin (mTOR) inhibitors have provided some benefits. However, disease progression has occurred in the majority of patients during disease course [2]. So there is a need for development of novel immunotherapeutic approaches for metastatic RCCs. Cancer-testis antigens (CTAs) as a group of tumor associated antigens (TAAs) are considered as appropriate cancer biomarkers as well as immunotherapeutic targets [3]. As their names imply their expression is almost limited to gametogenic tissues among normal tissues. However, they are aberrantly reactivated in several tumoral tissues [4]. As testis is regarded as an immune-privileged site, abnormal expression of CTAs in cancer is anticipated to elicit immune response. So
Submission Date: 05/14/2017Ａcceptance Date: 08/25/2017

these tumor antigens can be used in immunotherapeutic approaches to enhance immune response against cancer cells.

\section{Expression of CTAs in RCC \\ Armadillo Repeat Containing 3 (ARMC3)}

This CTA has been identified through screening of a testis cDNA library with serum from a patient with pancreatic ductal adenocarcinoma. Subsequently, its expression has been assessed in numerous cancer cell lines and tissues of different origins by reverse transcriptionPCR and Northern blot analyses. It has been shown to be expressed in 6 of 10 renal cancer cell lines (RCC6, RCC7, RCC8, Saito, ACHN, and A498) as well as 1 of 8 renal cell cancer tissues (13\%). Although spontaneous immune responses against this antigen have been detected in a variety of cancer patients, none of the assessed renal cancer patients were immunoreactive to this antigen [5].

\section{Cancer Antigen 1 (CAGE1)}

This CTA has been identified through screening of cDNA expression libraries with serum samples from lung cancer patients. This nuclear protein has been shown to have testis-specific expression pattern among normal tissues but high expression in numerous cancer tissues and cancer cell lines which implies its association with the tumorigenesis process. It has been shown to be expressed in 3 of 4 renal cancer cell lines [6]. In addition, its

\footnotetext{
Corresponding Author:

Dr. Soudeh Ghafouri-Fard

Department of Medical Genetics, Shahid Beheshti University of Medical Sciences, Tehran, Iran.

Email: s.ghafourifard@sbmu.ac.ir
} 
expression has been detected in 2/4 (50\%) of renal cancer cell lines assessed (Satio and RCC6) in another study [7]. The putative role of this protein is helicase activity. Notably, its expression in certain cancer cell lines has been activated by demethylating agents [8]. In addition, its oncogenic activity in cancer cell lines has been shown to be exerted through induction of cell cycle progression by up-regulation of AP-1- and E2F-dependent expression of cyclins D1 and E [9].

\section{Calreticulin 3 (CALR3)}

This CTA has been identified through representational differential analysis (RDA) and immunogenicity assessment. The encoded protein is localized in elongated spermatids of testis among normal tissues and in a wide range of cancer cell lines and tissues including renal cell carcinoma. Notably, a CALR3-originated peptide has been recognized as an HLA-A24- restricted T-cell epitope capable of induction of cytotoxic T cells (CTLs). So this CTA can induce both humoral and cellular immune responses [10].

\section{Coiled-coil domain containing 62 (CCDC62)}

This CTA has been identified through screening of a testicular cDNA library with a serum sample taken from a gastric adenocarcinoma patient with a history of vanished liver metastasis and tumor regression. Subsequently, its expression has been demonstrated in a variety of cancer samples including RCC. In addition, antibodies against CCDC62 have been detected in a subset of cancer patients but none of healthy donors [11].

\section{Cell division cycle associated gene 1 (CDCA1)}

This CTA has been firstly identified as a TAA in non-small cell lung cancer using cDNA microarray technology. Subsequently, its elevated expression levels have been detected in a significant number of patients with cholangiocellular cancer, urinary bladder cancer and RCC. Two CDCA1-derived peptides were able to induce peptide-reactive CTLs in HLA-A2.1 transgenic mice. Furthermore, in vitro stimulation of peripheral blood mononuclear cells with these peptides has resulted in induction of CTLs with the ability to kill CDCA1expressing tumor cells [12].

\section{CSAG family, member 2 (CSAG2)}

This CTA has been shown to be expressed in a variety of tumor samples including osteosarcoma, esophageal, gastric, hepatobiliary, lung and melanocytic cancers at the mRNA level and in ovarian cancer at the protein level. In addition, it has been shown to be involved in the mechanism of taxol resistance in ovarian cancer cells. Subsequently, its up-regulation has been detected in the taxol-resistant breast cancer cell line and the doxorubicin-resistant multiple myeloma cell lines. It has been shown to be expressed in $1 / 8(12 \%)$ of renal cancer cell lines assessed (SN12C) by Northern blot [13]. Spontaneous CSAG2-specific CD4+ T cells have been detected in cancer patients which can be used for assessment of spontaneous immune responses in patients with CSAG2-expressing tumors and for the development of immunotherapeutic modalities [14].

\section{Cancer Testis Antigen 47A1 (CT47A1)}

This CTA has been identified through an approach for exploration of transcript clusters that map to several locations on the chromosome and subsequent in silico analysis of their gene expression pattern which led to identification of five novel gene families with testisspecific expression and a high sequence similarity among family members. It has been shown to be expressed in SK-RCC-1 [15].

\section{CTAG1B (NY-ESO-1)}

Kermany et al. have detected NY-ESO-1 expression in 39 of $195(20 \%)$ of RCC tumors but none of benign kidney tissues. In addition, they demonstrated NY-ESO-1 expression in three of 6 renal cancer cell lines (A498, 704 and 786-0). They could induce NY-ESO-1 expression in all 6 cell lines by using hypomethylating agent. Besides, decitabine-treated A498 RCC cells produced elevated levels of IFN- $\gamma$ by activated T cells. Consequently, they suggested NY-ESO-1 as a potential target for immunotherapy in RCC [16]. Elevated expression levels of NY-ESO-1 have been demonstrated in a proportion of RCC samples in another study with higher expressions observed in metastatic samples compared with the corresponding primary tumors. NY-ESO-1 expression has been considerably higher in primary RCC samples compared with adjacent normal renal tissues and in clear cell carcinomas compared with papillary RCC [17].

\section{DnaJ Heat Shock Protein Family (Hsp40) Member B8 (DNAJB8)}

It has been shown to be expressed in ACHN, Caki-1, SMKTR2 and SMKTR3. In addition, it has been shown to participate in the cancer stem cell (CSC) phenotype in RCC. Its forced overexpression has elevated the percentage of side population (SP) cells characterizing CSCs in RCC cells and promoted their tumorigenic capacity. On the other hand, its down-regulation had the opposite effects in RCC lines. Most importantly, it has been shown to be an appropriate immunologic target, since compared with immunization with survivin, which was expressed in both CSCs and non-CSCs in RCC, immunization with Dnajb8 vaccine led to more robust antitumor effects [18].

\section{Interleukin 13 Receptor Subunit Alpha 2 (IL13RA2)}

It has been shown to be expressed in RCC, PM-RCC, ML-RCC, WS-RCC, HL-RCC, Caki-1, RC-2, U251 and IGROV-1 [19]. More recently, IL13RA2 has been identified as a potential molecule associated with the gained sunitinib-resistance in clear cell RCC (ccRCC). Elevated levels of IL13RA2 expression in primary ccRCC tumor have been shown to be associated with sunitinibresistant metastasis. Conversely, shRNA-mediated IL13RA2 silencing has attenuated the sunitinib resistance in Caki-1 cells [20]. 
Insulin like growth factor 2 mRNA binding protein 3 (IGF2BP3, IMP-3)

Its expression has been demonstrated in about one third of ccRCC specimens in a large cohort of patients. In addition, its expression has been with many oncogenic phenotypes of cancer cells including advanced stage and grade of primary tumors, coagulative tumor necrosis and sarcomatoid differentiation. More importantly, positive IMP3 expression was associated with a lower survival rate as well as higher risk of distant metastases [21]. PDZ binding kinase (PBK)

It is a serine/threonine kinase which is phosphorylated during mitosis and being overexpressed in several Burkitt's lymphoma cell lines and some patients' samples. Its expression profile implies its participation in the phenotype of highly proliferative malignant cells as well as normal fetal development. It has been shown to be expressed in RC298 as well [22].

Preferentially expressed antigen in melanoma (PRAME)

This CTA has been identified through stimulation of autologous lymphocytes with a melanoma metastasisderived cell line. This protein induces an HLA-A24restricted CTL clone that lysed these cells. Its expression has been demonstrated in a wide variety of cancer cells and also in some normal tissues, although in low amounts. It has been shown to be expressed in 24/ 58 (41\%) of renal cancer samples examined [23].

Sperm Protein Associated With The Nucleus, X Chromosome (SPANX) family

SPANX family proteins, specifically SPANX-C have been shown to be expressed in a variety of tumor cells including melanoma, RCC and cancers of bladder and prostate. High levels of SPANX-B expression have been found in melanoma and carcinomas of lung, ovary, colon, and breast. SPANX-B has been recognized by circulating T-cell precursors in normal subjects. SPANX-B-specific helper CD4+ and CTLs have been produced by expansion of these circulating T-cell precursors. The tumor cell killing function of these CTLS has been confirmed in HLAA2-expressing tumors [24].

\section{Discussion}

Expression and immunogenicity of CTAs has been assessed in a variety of malignancies [4, 25, 26]. In addition, there are a number of clinical trials evaluating the safety and efficacy of CTA-based immunotherapeutic approaches in cancer patients. However, data regarding expression of CTAs in RCC is scarce. For many of CTAs whose expression has been assessed in RCC cell lines, there is no supporting evidence for their expression in patients samples. However, in order to apply such results in clinical settings it is necessary to assess CTA expression in clinical samples of different stages and grades to obtain sufficient data regarding their role in the tumorigenesis process. NY-ESO-1 as the most studied CTA [27] has also been applied in clinical trials of RCC patients. For instance, RCC patients have been included in a phase 1 clinical trial of vaccine therapy with or without Sirolimus in treating patients with NY-ESO-1 expressing solid tumors (https://clinicaltrials.gov/ct2/show/NCT01522820) or a phase 1 clinical trial of TAA-Specific CTLs for Solid Tumors (TACTASOM) (https://clinicaltrials.gov/ct2/ show/NCT02239861). The latter study has targeted five common TAAs called NY-ESO-1, MAGEA4, PRAME, Survivin and SSX. The results of these studies and similar researches would help in identification of suitable targets for each cancer type as well as design of combinatory approach for treatment of cancers.

\section{References}

1. Ridge CA, Pua BB, Madoff DC. Epidemiology and staging of renal cell carcinoma. Seminars in interventional radiology. 2014;31(1):3-8.

2. Raman R, Vaena D. Immunotherapy in Metastatic Renal Cell Carcinoma: A Comprehensive Review. Biomed Res Int. 2015

3. Ghafouri-Fard S, Modarressi M-H. Cancer-testis antigens: potential targets for cancer immunotherapy. Arch Iran Med. 2009;12(4):395-404.

4. Ghafouri-Fard S, Abbasi A, Moslehi H, Faramarzi N, Taba Taba Vakili S, Mobasheri M, et al. Elevated expression levels of testis-specific genes TEX101 and SPATA19 in basal cell carcinoma and their correlation with clinical and pathological features. British Journal of Dermatology. 2010;162(4):772-9.

5. Okada T, Akada M, Fujita T, Iwata T, Goto Y, Kido K, et al. A novel cancer testis antigen that is frequently expressed in pancreatic, lung, and endometrial cancers. Clinical cancer research : an official journal of the American Association for Cancer Research. 2006;12(1):191-7.

6. Park S, Lim Y, Lee D, Cho B, Bang YJ, Sung S, et al. Identification and characterization of a novel cancer/testis antigen gene CAGE-1. Biochimica et biophysica acta. $2003 ; 1625(2): 173-82$.

7. Iwata T, Fujita T, Hirao N, Matsuzaki Y, Okada T, Mochimaru $\mathrm{H}$, et al. Frequent immune responses to a cancer/testis antigen, CAGE, in patients with microsatellite instabilitypositive endometrial cancer. Clinical cancer research : an official journal of the American Association for Cancer Research. 2005;11(10):3949-57.

8. Kim Y, Jeoung D. Role of CAGE, a novel cancer/testis antigen, in various cellular processes, including tumorigenesis, cytolytic T lymphocyte induction, and cell motility. Journal of microbiology and biotechnology. 2008;18(3):600-10.

9. Por E, Byun HJ, Lee EJ, Lim JH, Jung SY, Park I, et al. The Cancer/Testis Antigen CAGE with Oncogenic Potential Stimulates Cell Proliferation by Up-regulating Cyclins D1 and $\mathrm{E}$ in an AP-1-and E2F-dependent Manner. J Biol Chem. 2010;285(19):14475-85.

10. Hayashi E, Matsuzaki Y, Hasegawa G, Yaguchi T, Kurihara $\mathrm{S}$, Fujita $\mathrm{T}$, et al. Identification of a novel cancer-testis antigen CRT2 frequently expressed in various cancers using representational differential analysis. Clinical cancer research : an official journal of the American Association for Cancer Research. 2007;13(21):6267-74.

11. Domae S, Nakamura Y, Nakamura Y, Uenaka A, Wada H, Nakata M, et al. Identification of CCDC62-2 as a novel cancer/testis antigen and its immunogenicity. International journal of cancer. 2009;124(10):2347-52. 
12. Harao M, Hirata S, Irie A, Senju S, Nakatsura T, Komori $\mathrm{H}$, et al. HLA-A2-restricted CTL epitopes of a novel lung cancer-associated cancer testis antigen, cell division cycle associated 1 , can induce tumor-reactive CTL. International journal of cancer. 2008;123(11):2616-25.

13. Duan Z, Feller AJ, Toh HC, Makastorsis T, Seiden MV. TRAG-3, a novel gene, isolated from a taxol-resistant ovarian carcinoma cell line. Gene. 1999;229(1-2):75-81.

14. Janjic B, Andrade P, Wang XF, Fourcade J, Almunia $\mathrm{C}$, Kudela $\mathrm{P}$, et al. Spontaneous CD4+ T cell responses against TRAG-3 in patients with melanoma and breast cancers. Journal of immunology (Baltimore, Md : 1950). 2006;177(4):2717-27.

15. Chen YT, Iseli C, Venditti CA, Old LJ, Simpson AJ, Jongeneel CV. Identification of a new cancer/testis gene family, CT47, among expressed multicopy genes on the human X chromosome. Genes, chromosomes \& cancer. 2006;45(4):392-400.

16. Kermany MH, Heit L, Wald A, Schwaab T. MP29-17 CANCER/TESTIS ANTIGEN NY-ESO-1-A POTENTIAL TARGET FOR IMMUNOTHERAPY IN RENAL CELL CARCINOMA (RCC). The Journal of Urology. 2014;191(4):e311.

17. Giesen E, Jilaveanu LB, Parisi F, Kluger Y, Camp RL, Kluger HM. NY-ESO-1 as a potential immunotherapeutic target in renal cell carcinoma. Oncotarget. 2014;5(14):5209-17.

18. Nishizawa S, Hirohashi Y, Torigoe T, Takahashi A, Tamura Y, Mori T, et al. HSP DNAJB8 controls tumor-initiating ability in renal cancer stem-like cells. Cancer research. 2012;72(11):2844-54.

19. Murata T, Obiri NI, Debinski W, Puri RK. Structure of IL-13 receptor: analysis of subunit composition in cancer and immune cells. Biochemical and biophysical research communications. 1997;238(1):90-4.

20. Shibasaki N, Yamasaki T, Kanno T, Arakaki R, Sakamoto H, Utsunomiya N, et al. Role of IL13RA2 in Sunitinib Resistance in Clear Cell Renal Cell Carcinoma. PloS one. 2015;10(6):e0130980.

21. Hoffmann NE, Sheinin Y, Lohse CM, Parker AS, Leibovich $\mathrm{BC}$, Jiang Z, et al. External validation of IMP3 expression as an independent prognostic marker for metastatic progression and death for patients with clear cell renal cell carcinoma. Cancer. 2008;112(7):1471-9.

22. Simons-Evelyn M, Bailey-Dell K, Toretsky JA, Ross DD, Fenton R, Kalvakolanu D, et al. PBK/TOPK is a novel mitotic kinase which is upregulated in Burkitt's lymphoma and other highly proliferative malignant cells. Blood cells, molecules \& diseases. 2001;27(5):825-9.

23. Ikeda H, Lethe B, Lehmann F, van Baren N, Baurain JF, de Smet $\mathrm{C}$, et al. Characterization of an antigen that is recognized on a melanoma showing partial HLA loss by CTL expressing an NK inhibitory receptor. Immunity. 1997;6(2):199-208.

24. Almanzar G, Olkhanud PB, Bodogai M, Dell'Agnola C, Baatar D, Hewitt SM, et al. Sperm-derived SPANX-B is a clinically relevant tumor antigen that is expressed in human tumors and readily recognized by human CD4+ and CD8+ T cells. Clinical Cancer Research. 2009;15(6):1954-63.

25. Ghafouri-Fard S, Modarressi MH, Yazarloob F. Expression of testis-specific genes, TEX101 and ODF4, in chronic myeloid leukemia and evaluation of TEX101 immunogenicity. Annals of Saudi medicine. 2012;32(3).

26. Dianatpour M, Mehdipour P, Nayernia K, Mobasheri M-B, Ghafouri-Fard S, Savad S, et al. Expression of testis specific genes TSGA10, TEX101 and ODF3 in breast cancer. Iranian Red Crescent Medical Journal. 2012;14(11):722.
27. Esfandiary A, Ghafouri-Fard S. New York esophageal squamous cell carcinoma-1 and cancer immunotherapy. immunotherapy. 2015;7(4):411-39.

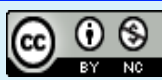

This work is licensed under a Creative Commons AttributionNon Commercial 4.0 International License. 